Bull. Korean Math. Soc. 49 (2012), No. 3, pp. 511-515

http://dx.doi.org/10.4134/BKMS.2012.49.3.511

\title{
PRIME RADICALS IN UP-MONOID RINGS
}

\author{
Jeoung Soo Cheon And Jin-A Kim
}

\begin{abstract}
We first show that the semiprimeness, primeness, and reducedness can go up to up-monoid rings. By these results we can compute the lower nilradicals of up-monoid rings, from which the well-known fact of Amitsur and McCoy for the polynomial rings can be extended to up-monoid rings.
\end{abstract}

A monoid $G$ is called a unique product monoid (simply, up-monoid) if given any two nonempty finite subsets $A$ and $B$ of $G$ there exists at least one $c \in G$ that has a unique representation in the form $c=a b$ with $a \in A$ and $b \in B$. A group is called a up-group if it satisfies the preceding condition. The study of up-monoids has important roles in group theory and ring theory (see [6], [7] for more details). Group algebras of up-groups are extensively observed relating to the zero divisor problem (see [7]). These lead us to study the basic structure of monoid rings of up-monoids relating to the (semi)primeness and reducedness. Many other relevant results can be found in [1] and [2].

Throughout this note each ring is associative and possibly without identity. A ring is called reduced if it has no nonzero nilpotent elements. A ring is called semiprime if the prime radical is zero. Reduced rings are clearly semiprime and note that a commutative ring is semiprime if and only if it is reduced.

Let $R$ be a reduced ring. Then with the help of [5] we have that if $x_{1} x_{2} \cdots x_{n}$ $=0$ for $x_{i} \in R$, then $x_{\sigma(1)} x_{\sigma(2)} \cdots x_{\sigma(n)}=0$ for any permutation $\sigma$ of $\{1,2, \ldots$, $n$ \}. We will use this result freely in the process. The following is obtained by applying relevant results in [3]. But here we obtain our result through direct computations, watching what elements are doing.

Theorem 1. Let $R$ be a ring and $G$ a up-monoid. Write $S=R G$.

(1) $R$ is semiprime if and only if so is $S$.

(2) $R$ is prime if and only if so is $S$.

(3) $R$ is reduced if and only if so is $S$.

(4) $R$ is a domain if and only if so is $S$.

Received December 24, 2010.

2010 Mathematics Subject Classification. Primary 16N40, 16N60.

Key words and phrases. up-monoid ring, lower nilradical, upper nilradical, (semi)prime ring, reduced ring. 
Proof. (1) Let $R$ be semiprime. Assume on the contrary that there exists $0 \neq f=\sum_{i=1}^{n} a_{i} g_{i} \in S$ satisfying $f S f=0$. We can assume that every $a_{i}$ is nonzero. From $f S f=0$, we get $f R f=0$. Since $G$ is a up-monoid, there exists a unique product $g_{i} g_{j}$, obtaining $a_{i} R a_{j}=0$. So $f R f=0$ implies

$0=a_{i} r f R a_{i} r f=\left(\cdots+a_{i} r a_{j-1}+a_{i} r a_{j+1}+\cdots\right) R\left(\cdots+a_{i} r a_{j-1}+a_{i} r a_{j+1}+\cdots\right)$, where $r$ is arbitrary in $R$.

Set $b_{s}=a_{i} r a_{s}$ and $f_{1}=\sum_{s=1}^{n_{1}} b_{s} g_{s}$. Then we get $f_{1} R f_{1}=0$ with $f_{1}=a_{i} r f$. Assuming $f_{1}=0$ for all $r \in R$, we get $a_{i} R a_{i}=0$ but this induces a contradiction since $R$ is semiprime and $a_{i}$ is nonzero (hence $a_{i} R a_{i} \neq 0$ ). Thus we have $f_{1} R f_{1}=0$ with $f_{1}=a_{i} r f \neq 0$ for some $r \in R$. We can also assume that every $b_{s}$ is nonzero. Note $n_{1}<n$.

We repeat the preceding computation once more for completeness. Since $G$ is a up-monoid, there exists a unique product $g_{v} g_{w}$, obtaining $b_{v} R b_{w}=0$. Thus $f_{1} R f_{1}=0$ implies

$$
\begin{aligned}
0 & =b_{v} x f_{1} R b_{v} x f_{1} \\
& =\left(\cdots+b_{v} x b_{w-1}+b_{v} x b_{w+1}+\cdots\right) R\left(\cdots+b_{v} x b_{w-1}+b_{v} x b_{w+1}+\cdots\right),
\end{aligned}
$$

where $x$ is arbitrary in $R$. Here since $R$ is semiprime and $b_{v} \neq 0$, we have $b_{v} R b_{v} \neq 0$ and so $f_{2}=b_{v} x f_{1} \neq 0$ for some $x \in R$. Letting $f_{2}=\sum_{t=1}^{n_{2}} c_{t} g_{t}$ with $c_{t} \in R$, we get $n_{2}<n_{1}<n$.

Proceeding in this manner, we finally reach $f_{k}=\left(d g_{h}\right) R\left(d g_{h}\right)=0$ with $0 \neq d \in R$ and $g_{h} \in\left\{g_{1}, \ldots, g_{n}\right\}$, entailing $d R d=0$. But since $R$ is semiprime, we have $d=0$, a contradiction. Therefore $f S f=0$ implies $f=0$.

Next suppose that $a R a=0$ for $a \in R$. Then $a S a=0$, and so if $S$ is semiprime, then $a=0$.

(2) Let $R$ be prime. Suppose that there exist $f=\sum_{i=1}^{m} a_{i} g_{i}, g=\sum_{j=1}^{n} b_{j} h_{j}$ $\in S \backslash\{0\}$ satisfying $f S g=0$. We can assume that $a_{i}, b_{j} \in R \backslash\{0\}$ for all $i, j$. From $f S g=0$, we get $f R g=0$. Since $G$ is a up-monoid, there exists a unique product $g_{i} h_{j}$, obtaining $a_{i} R b_{j}=0$. Then since $R$ is prime, we get $a_{i}=0$ or $b_{j}=0$, a contradiction.

Next let $a R b=0$ for $a, b \in R$. Then $a S b=0$, and so if $S$ is prime, then $a=0$ or $b=0$.

(3) It suffices to show the necessity since the reducedness is preserved by subrings. We apply the proof of (1). Let $R$ be reduced. Assume on the contrary that there exists $0 \neq f=\sum_{i=1}^{n} a_{i} g_{i} \in S$ satisfying $f^{2}=0$. We can assume that every $a_{i}$ is nonzero. Since $G$ is a up-monoid, there exists a unique product $g_{i} g_{j}$, obtaining $a_{i} a_{j}=0$. Since $R$ is reduced, $x y=0$ implies $y x=0$ for $x, y \in R$. We will use this fact freely. From $a_{i} a_{j}=0$, we get $a_{j} a_{i}=0$ and so

$$
0=a_{i} f f a_{i}=\left(\cdots+a_{i} a_{j-1}+a_{i} a_{j+1}+\cdots\right)\left(\cdots+a_{j-1} a_{i}+a_{j+1} a_{i}+\cdots\right) .
$$

But since $R$ is reduced, $a_{i} \neq 0$ implies $a_{i}^{2} \neq 0$ and so $a_{i} f, f a_{i}$ are both nonzero. Put $f_{11}=a_{i} f$ and $f_{12}=f a_{i}$. Note that the number of nonzero terms in $f_{1 \ell}$, 
say $n_{1 \ell}$, is less than $n$ for $\ell=1,2$. Since $R$ is reduced, $n_{11}=n_{12}$ and $a_{i} a_{\alpha}=0$ $\Leftrightarrow a_{\alpha} a_{i}=0$ for $\alpha \in\{1, \ldots, n\}$.

Since $G$ is a up-monoid, there exists a unique product $g_{s} g_{t}$, obtaining $a_{i} a_{s} a_{t} a_{i}=0$ (here we can assume that $a_{i} a_{s}$ and $a_{t} a_{i}$ are both nonzero). Then $a_{i} a_{s} a_{t}=0, a_{t} a_{i} a_{s}=0$ since $R$ is reduced, and so

$$
\begin{aligned}
0 & =a_{i} a_{s} f f a_{i} a_{s} \\
& =\left(\cdots+a_{i} a_{s} a_{t-1}+a_{i} a_{s} a_{t+1}+\cdots\right)\left(\cdots+a_{t-1} a_{i} a_{s}+a_{t+1} a_{i} a_{s}+\cdots\right) .
\end{aligned}
$$

But since $R$ is reduced, $a_{i} a_{s} \neq 0$ implies $\left(a_{i} a_{s}\right)^{2} \neq 0$ and so $a_{i} a_{s} f, f a_{i} a_{s}$ are both nonzero. Put $f_{21}=a_{i} a_{s} f$ and $f_{22}=f a_{i} a_{s}$. Then each $f_{2 \ell}$ is nonzero. Note that the number of nonzero terms in $f_{2 \ell}$, say $n_{2 \ell}$, is less than $n_{1 \ell}$ for $\ell=1,2$. Since $R$ is reduced, $n_{21}=n_{22}$ and $a_{i} a_{s} a_{\beta}=0 \Leftrightarrow a_{\beta} a_{i} a_{s}=0$ for $\beta \in\{1, \ldots, n\}$.

Proceeding in this manner, we finally obtain $a_{\alpha 1}, \ldots, a_{\alpha k} \in\left\{a_{1}, \ldots, a_{n}\right\}$ such that

$$
0=a_{\alpha 1} \cdots a_{\alpha k} f f a_{\alpha 1} \cdots a_{\alpha k} \text { with } a_{\alpha 1} \cdots a_{\alpha k} f, f a_{\alpha 1} \cdots a_{\alpha k} \in R \backslash 0 .
$$

Say $a_{\alpha 1} \cdots a_{\alpha k} f=a_{\alpha 1} \cdots a_{\alpha k} d$ for some $d \in R$. In the process, we get $a_{\alpha 1} \cdots a_{\alpha h} a_{v}=0$ if and only if $a_{v} a_{\alpha 1} \cdots a_{\alpha h}=0$ for each $h \leq k$. Whence we also have

$$
a_{\alpha 1} \cdots a_{\alpha k} d=a_{\alpha 1} \cdots a_{\alpha k} f=d a_{\alpha 1} \cdots a_{\alpha k},
$$

entailing $\left(a_{\alpha 1} \cdots a_{\alpha k} d\right)^{2}=0$, and so since $R$ is reduced, $a_{\alpha 1} \cdots a_{\alpha k} d=0$, a contradiction. Therefore $f^{2}=0$ implies $f=0$.

(4) The proof is similar to (2). It suffices to show the necessity since any subring of a domain is also a domain. Let $R$ be a domain. Suppose that there exist $f=\sum_{i=1}^{m} a_{i} g_{i}, g=\sum_{j=1}^{n} b_{j} h_{j} \in S \backslash\{0\}$ satisfying $f g=0$. We can assume that $a_{i}, b_{j} \in R \backslash\{0\}$ for all $i, j$. Since $G$ is a up-monoid, there exists a unique product $g_{i} h_{j}$, obtaining $a_{i} b_{j}=0$. Then since $R$ is a domain, we get $a_{i}=0$ or $b_{j}=0$, a contradiction.

Let $X$ be a set of commuting indeterminates over a ring $R$. It is well-known that the set of all finite products of indeterminates in $X$ with 1 forms a upmonoid. So we get the following well-known results for the polynomial rings from Theorem 1. The polynomial ring with $X$ over $R$ is denoted by $R[X]$.

Corollary 2. (1) [4, Proposition 10.18] A ring $R$ is semiprime if and only if so is $R[X]$.

(2) $[4$, Proposition 10.18] $A$ ring $R$ is prime if and only if so is $R[X]$.

(3) $A$ ring $R$ is reduced if and only if so is $R[X]$.

(4) $A$ ring $R$ is a domain if and only if so is $R[X]$.

We next compute the lower nilradicals of the monoid rings. The lower nilradical (i.e., prime radical) of a ring $A$ is denoted by $N_{*}(A)$.

Theorem 3. Let $R$ be a ring and $G$ a up-monoid. Then $N_{*}(R G)=N_{*}(R) G$. 
Proof. We apply the process of Amitsur and McCoy [4, Theorem 10.19]. Let $N=N_{*}(R)$. Note $\frac{R G}{N G} \cong \frac{R}{N} G$. Since $\frac{R}{N} G$ is semiprime by Theorem $1(1)$, we have that $N G$ is a semiprime ideal of $R G$, entailing $N_{*}(R G) \subseteq N G$. For the converse, let $P$ be a prime ideal of $R G$. Let $Q=P \cap R$ and suppose $a R b \subseteq Q$ for $a, b \in R$. Then $a R G b \subseteq P$ and so $a \in P$ or $b \in P$ (hence $a \in Q$ or $b \in Q$ ), concluding that $Q$ is a prime ideal of $R$. Thus $Q G$ is a prime ideal of $R G$ by Theorem $1(2)$ from $\frac{R G}{Q G} \cong \frac{R}{Q} G$. It then follows

$$
N G \subseteq Q G \subseteq P
$$

since $Q \subseteq P$, obtaining $N G \subseteq N_{*}(R G)$.

Corollary 4 ([4, Theorem 10.19] (Amitsur, McCoy)). Let $R$ be a ring. Then $N_{*}(R[X])=N_{*}(R)[X]$.

Denote the set of all nilpotent elements in a ring $A$ by $N(A)$.

Theorem 5. Let $R$ be a ring and $G$ a up-monoid. Then $N_{*}(R)=N(R)$ if and only if $N_{*}(R G)=N(R G)$.

Proof. $N_{*}(R G)=N_{*}(R) G$ by Theorem 3, and so if $N_{*}(R G)=N(R G)$, then

$$
N(R)=R \cap N(R G)=R \cap N_{*}(R G)=R \cap N_{*}(R) G=N_{*}(R) .
$$

Conversely let $N(R)=N_{*}(R)$. Then by Theorem 3, $N_{*}(R G)=N_{*}(R) G=$ $N(R) G$. Since $R / N(R)$ is reduced, $\frac{R}{N(R)} G \cong \frac{R G}{N(R) G}$ is reduced by Theorem $1(3)$, entailing $N(R G) \subseteq N(R) G=N_{*}(R) G$. But by Theorem 3, we get $N(R) G=N_{*}(R) G=N_{*}(R G) \subseteq N(R G)$ and so $N_{*}(R G)=N(R G)$.

In the following we can see various kinds of up-groups (hence up-monoids). The ring of Laurent polynomials in $x$, coefficients in a ring $R$, consists of all formal sums $\sum_{i=k}^{n} m_{i} x^{i}$ with obvious addition and multiplication, where $m_{i} \in$ $R$ and $k, n$ are (possibly negative) integers; denote it by $R\left[x ; x^{-1}\right]$.

Proposition 6. Let $R$ be a ring and $G$ a group. Then any of the following rings $R G$ satisfies Theorem 1, Theorem 3, and Theorem 5:

(1) $R G=R\left[x ; x^{-1}\right]$.

(2) $R G$ when $G$ is right or left ordered group.

(3) $R G$ when $G$ has a normal subgroup $H$ such that both $H$ and $G / H$ are up-groups.

(4) $R G$ when every finitely generated nonidentity subgroup of $G$ can be mapped homomorphically onto a nonidentity up-group.

(5) $R G$ when $G$ has a finite subnormal series $\langle 1\rangle=G_{0} \leq G_{1} \leq \cdots \leq G_{n}=G$ such that each $G_{i+1} / G_{i}$ is a torsion-free abelian group.

(6) $R G$ when $G$ is a torsion-free nilpotent group.

Proof. (1) $G=\left\{\ldots, x^{-2}, x^{-1}, 1, x, x^{2}, \ldots\right\}$ is obviously a up-group, and $R G=$ $R\left[x ; x^{-1}\right] . G$ in (2) is a up-group by [7, Lemma 13.1.7]. $G$ in (3) and (4) is a up-group by [7, Lemma 13.1.8]. $G$ in (5) and (6) is a up-group by [7, Lemmas 13.1.6 and 13.1.7]. 
In Section 2 in [7, Chapter 13], we can find various kinds of (one-sided) ordered groups.

The upper nilradical (i.e., the sum of all nil ideals) of a ring $A$ is denoted by $N^{*}(A)$. Note $N_{*}(A) \subseteq N^{*}(A) \subseteq N(A)$.

As contrasted with Theorems 3 and 5 , we have negative situations for the upper nilradicals. With the help of the computations of Smoktunowicz [8], there exists a ring $R$ with $N^{*}(R)=N(R)$ but $N^{*}(R[X]) \varsubsetneqq N(R[X]) \varsubsetneqq N^{*}(R)[X]$.

Acknowledgments. The authors would like to thank the referee for his or her careful reading of the text. They also thank Professor Yang Lee for providing valuable guidance and suggestions throughout the course of this research.

\section{References}

[1] V. Camillo, C. Y. Hong, N. K. Kim, Y. Lee, and P. P. Nielsen, Nilpotent ideals in polynomial and power series rings, Proc. Amer. Math. Soc. 138 (2010), no. 5, 16071619.

[2] C. Y. Hong, N. K. Kim, and Y. Lee, Extensions of McCoy's theorem, Glasg. Math. J. 52 (2010), no. 1, 155-159.

[3] E. Jespersa, J. Krempa, and E. R. Puczylowski, On radicals of graded rings, Comm. Algebra. 10 (1982), 1849-1854.

[4] T. Y. Lam, A First Course in Noncommutative Rings, Springer-Verlag, New York, 1991.

[5] J. Lambek, On the representations of modules by sheaves of factor modules, Canad. Math. Bull. 14 (1971), 359-368.

[6] J. Okninski, Semigroup Algebras, Marcel Dekker, New York, 1991.

[7] D. S. Passman, The Algebraic Structure of Group Rings, Wiley, New York, 1977.

[8] A. Smoktunowicz, Polynomial rings over nil rings need not be nil, J. Algebra 233 (2000), no. $2,427-436$

JeOung Soo Cheon

Department of Mathematics

Pusan National University

PusAn 609-735, Korea

E-mail address: jeoungsoo@pusan.ac.kr

JIN-A KIM

Department of Mathematics

Pusan National University

PUSAN 609-735, KoreA

E-mail address: sky1030k@hanmail.net 